Publ. Mat. 47 (2003), 451-457

\title{
CENTRE-BY-METABELIAN GROUPS WITH A CONDITION ON INFINITE SUBSETS
}

\author{
NADIR TRABELSI
}

\begin{abstract}
In this note, we consider some combinatorial conditions on infinite subsets of groups and we obtain in terms of these conditions some characterizations of the classes $\mathcal{L}\left(\mathcal{N}_{k}\right) \mathcal{F}$ and $\mathcal{F} \mathcal{L}\left(\mathcal{N}_{k}\right)$ for the finitely generated centre-by-metabelian groups, where $\mathcal{L}\left(\mathcal{N}_{k}\right)$ (respectively, $\mathcal{F}$ ) denotes the class of groups in which the normal closure of each element is nilpotent of class at most $k$ (respectively, finite groups).
\end{abstract}

\section{Introduction and results}

Following a question of Erdös, B. H. Neumann proved in [13] that a group is centre-by-finite if, and only if, every infinite subset contains a commuting pair of distinct elements. Since this result, problems of similar nature have been the object of many papers (for example [1], $[\mathbf{2}],[\mathbf{3}],[\mathbf{4}],[\mathbf{5}],[\mathbf{9}],[\mathbf{1 1}],[\mathbf{1 0}],[\mathbf{1 6}],[\mathbf{1 7}])$. We present here some further results of the same type.

Let $k$ be a fixed positive integer. Denote by $E_{k}^{*}$ the class of groups such that for every infinite subset $X$ there exist two distinct elements $x, y$ in $X$, and integers $t_{0}, t_{1}, \ldots, t_{k}$ depending on $x, y$, and satisfying $\left[z_{0}^{t_{0}}, z_{1}^{t_{1}}, \ldots, z_{k}^{t_{k}}\right]=1$, where $z_{i} \in\{x, y\}$ for every $i \in\{0,1, \ldots, k\}$ and $z_{0} \neq z_{1}$. Denote also by $E_{k}^{\#}$ the class of groups $G \in E_{k}^{*}$ for which the integers $t_{0}, \ldots, t_{k}$ belong to $\{-1,1\}$. In [3] , it is proved that if $G$ is a finitely generated soluble group in the class $E_{k}^{*}$ (respectively $E_{k}^{\#}$ ), then there is an integer $c$, depending only on $k$, such that $G$ is in $\mathcal{N}_{c} \mathcal{F}$ (respectively $\mathcal{F} \mathcal{N}_{c}$ ); where $\mathcal{N}_{c}$ and $\mathcal{F}$ denote respectively the class of nilpotent groups of class at most $c$ and the class of finite groups. In [3], it is also proved that a finitely generated metabelian group $G$ is in $E_{k}^{*}$ (respectively $E_{k}^{\#}$ ) if, and only if, $G$ belongs to $\mathcal{N}_{k} \mathcal{F}$ (respectively $\mathcal{F} \mathcal{N}_{k}$ ); and it is 2000 Mathematics Subject Classification. 20F16, $20 \mathrm{~F} 45$.

Key words. Engel conditions, finitely generated soluble groups, Levi classes, nilpotent groups. 
observed that these results are not true if the derived length of $G$ is $\geq 3$. Among the examples cited, which are due to Newman [14] (see also [2]), there is a finitely generated torsion-free nilpotent group $G$ of class 4 , of derived length 3 , and whose 2 -generated subgroups are nilpotent of class at most 3. So $G$ is a finitely generated centre-by-metabelian group which belongs to $E_{3}^{*}$ (respectively $E_{3}^{\#}$ ) and such that $G \notin \mathcal{N}_{3} \mathcal{F}$ (respectively $\left.G \notin \mathcal{F} \mathcal{N}_{3}\right)$. Note that if a group belongs to $\mathcal{N}_{k}$, then it is in $\mathcal{L}\left(\mathcal{N}_{k-1}\right)$, where $\mathcal{L}\left(\mathcal{N}_{k-1}\right)$ denotes the class of groups in which the normal closure of each element is nilpotent of class at most $k-1$. Considering this weaker condition we are able to prove the following results:

Theorem 1.1. A finitely generated centre-by-metabelian group $G$ is in $E_{k+1}^{*}$ if, and only if, $G$ belongs to $\mathcal{L}\left(\mathcal{N}_{k}\right) \mathcal{F}$.

Theorem 1.2. A finitely generated centre-by-metabelian group $G$ is in $E_{k+1}^{\#}$ if, and only if, $G$ belongs to $\mathcal{F} \mathcal{L}\left(\mathcal{N}_{k}\right)$. In particular, a torsionfree centre-by-metabelian group $G$ is in $E_{k+1}^{\#}$ if, and only if, $G$ belongs to $\mathcal{L}\left(\mathcal{N}_{k}\right)$.

In [7], it is proved that a metabelian group $G$ is $(k+1)$-Engel if, and only if, $G$ belongs to $\mathcal{L}\left(\mathcal{N}_{k}\right)$. Morse [12] extended this result to a certain class of soluble groups of derived length $\leq 5$ which contains the centreby-metabelian groups. So our theorems improve Morse's result for the centre-by-metabelian groups.

Denote by $\mathcal{B}_{k}^{*}$ the class of groups such that every infinite subset contains an element $x$ such that $\langle x\rangle$ is subnormal of defect $k$. It is proved in [8, Corollary 2.5] that a metabelian non-torsion group is a $k$-Baer group (that is every cyclic subgroup of $G$ is subnormal of defect $k$ ) if, and only if, $G$ is a $k$-Engel group. Here, using Theorem 1.2, we shall improve this result with the following:

Theorem 1.3. Let $G$ be a finitely generated centre-by-metabelian group. If $G$ is in $\mathcal{B}_{k}^{*}$, then $G$ is finite-by-(k-Engel). In particular, a torsion-free centre-by-metabelian group $G$ belongs to $\mathcal{B}_{k}^{*}$ if, and only if, $G$ is $k$-Engel.

\section{Proof of the results}

Lemma 2.1. Let $G$ be a finitely generated torsion-free nilpotent group of class at most $k+1$. If $G$ belongs to $E_{k}^{*}$, then $G$ is a $k$-Engel group. 
Proof: Let $G$ be a group in $E_{k}^{*}$ and assume that $G$ is not $k$-Engel. Therefore there exist $x, y$ in $G$ such that $\left[x,{ }_{k} y\right] \neq 1$. The group $G$, being a finitely generated torsion-free nilpotent group, is a residually finite $p$-group for every prime $p$. So $G$ has a normal subgroup $N$ such that $\left[x,{ }_{k} y\right] \notin N$ and $|G / N|=p^{r}$ for some positive integer $r$. Considering the infinite subset $\left\{x^{p^{r+i}} y: i\right.$ integer $\}$, there are integers $n, m, t_{0}, t_{1}, \ldots, t_{k}$ such that $\left[z_{0}^{t_{0}}, z_{1}^{t_{1}}, \ldots, z_{k}^{t_{k}}\right]=1$, where $z_{i} \in\left\{x^{p^{r+n}} y, x^{p^{r+m}} y\right\}, n \neq m$ and $z_{0} \neq z_{1}$. Since $G$ is nilpotent of class at most $k+1$, the commutator $\left[z_{0}^{t_{0}}, z_{1}^{t_{1}}, \ldots, z_{k}^{t_{k}}\right]$ is linear in each argument $[\mathbf{1}$, Lemma 1], so we get that $\left[z_{0}, z_{1}, \ldots, z_{k}\right]^{t_{0} t_{1} \ldots t_{k}}=1$, and therefore $\left[z_{0}, z_{1}, \ldots, z_{k}\right]=1$ since $G$ is torsion-free. Put $z_{0}=x^{p^{r+s_{0}}} y$ and $z_{1}=x^{p^{r+s_{1}}} y$, where $s_{0} \neq s_{1} \in\{m, n\}$. So

$$
\begin{aligned}
1 & =\left[z_{0}, z_{1}, \ldots, z_{k}\right]=\left[\left[x^{p^{r+s_{0}}} y, x^{p^{r+s_{1}}} y\right], z_{2}, \ldots, z_{k}\right] \\
& =\left[\left[x^{\left(p^{r+s_{0}}-p^{r+s_{1}}\right)}, y\right]^{z_{1}}, z_{2}, \ldots, z_{k}\right]=\left[x^{\left(p^{r+s_{0}}-p^{r+s_{1}}\right)}, y, z_{2}, \ldots, z_{k}\right]^{z_{1}} .
\end{aligned}
$$

Hence

$$
1=\left[x^{\left(p^{r+s_{0}}-p^{r+s_{1}}\right)}, y, z_{2}, \ldots, z_{k}\right]=\left[x, y, z_{2}, \ldots, z_{k}\right]^{\left(p^{r+s_{0}}-p^{r+s_{1}}\right)} .
$$

Thus $\left[x, y, z_{2}, \ldots, z_{k}\right]=1$ as $G$ is torsion-free and $s_{0} \neq s_{1}$. Consequently $\left[x, y, z_{2}, \ldots, z_{k}\right] N=N$. Now $x^{p^{r+n}}, x^{p^{r+m}} \in N$, so $z_{i} N=y N$. It follows that $\left[x,{ }_{k} y\right] N=N$; this means that $\left[x,{ }_{k} y\right] \in N$, a contradiction which completes the proof.

It is proved in [12, Theorem 1] that if $G$ is nilpotent of class at most $k+2$, then $G$ is $(k+1)$-Engel if and only if $G \in \mathcal{L}\left(\mathcal{N}_{k}\right)$. So combining this result and Lemma 2.1, we have the following consequence:

Lemma 2.2. Let $G$ be a finitely generated nilpotent group of class at most $k+2$. If $G$ is in $E_{k+1}^{*}$, then $G$ belongs to $\mathcal{F} \mathcal{L}\left(\mathcal{N}_{k}\right)$. In particular, a torsion-free nilpotent group $G$ of class at most $k+2$ is in $E_{k+1}^{*}$ if, and only if, $G$ belongs to $\mathcal{L}\left(\mathcal{N}_{k}\right)$.

Proof: Let $G$ be a finitely generated nilpotent group of class at most $k+2$ and suppose that $G$ is in $E_{k+1}^{*}$. Then $T$, the torsion subgroup of $G$, is finite and $G / T$ is a finitely generated torsion-free group of nilpotency class at most $k+2$ which belongs to $E_{k+1}^{*}$. It follows, from Lemma 2.1, that $G / T$ is a $(k+1)$-Engel group, and by [12, Theorem 1], $G / T$ belongs to $\mathcal{L}\left(\mathcal{N}_{k}\right)$. Hence, $G$ is in $\mathcal{F} \mathcal{L}\left(\mathcal{N}_{k}\right)$; as claimed. 
Now, we suppose that $G$ is a torsion-free group of nilpotency class at most $k+2$ which belongs to $E_{k+1}^{*}$ and let $x, y_{1}, \ldots, y_{k+1} \in G$. Then $H=\left\langle x, y_{1}, \ldots, y_{k+1}\right\rangle$ is a finitely generated group of nilpotency class at most $k+2$ which belongs to $E_{k+1}^{*}$. It follows, from the first part of the proof, that $H$ is in $\mathcal{F} \mathcal{L}\left(\mathcal{N}_{k}\right)$. So $H$ is in $\mathcal{L}\left(\mathcal{N}_{k}\right)$ since it is torsion-free. Hence, $\left[x^{y_{1}}, \ldots, x^{y_{k+1}}\right]=1$, and this means that $G$ belongs to $\mathcal{L}\left(\mathcal{N}_{k}\right)$.

Clearly, any group in $\mathcal{L}\left(\mathcal{N}_{k}\right)$ is $(k+1)$-Engel, so it belongs to $E_{k+1}^{*}$.

Proof of Theorem 1.1: Let $G$ be a finitely generated centre-by-metabelian group in $E_{k+1}^{*}$. So $G / Z(G)$ is a finitely generated metabelian group in $E_{k+1}^{*}$. Therefore, by [3, Theorem 1.3], $G / Z(G)$ is in $\mathcal{N}_{k+1} \mathcal{F}$. Hence, $G$ belongs to $\mathcal{N}_{k+2} \mathcal{F}$. Since finitely generated nilpotent groups are (torsion-free)-by-finite [15, 5.4.15(i)], $G$ has a normal subgroup $H$, of finite index such that $H$ is a torsion-free nilpotent group of class at most $k+2$ which belongs to $E_{k+1}^{*}$. It follows, by Lemma 2.2 , that $H$ is in $\mathcal{L}\left(\mathcal{N}_{k}\right)$; so $G$ belongs to $\mathcal{L}\left(\mathcal{N}_{k}\right) \mathcal{F}$.

Conversely, suppose that $G$ is in $\mathcal{L}\left(\mathcal{N}_{k}\right) \mathcal{F}$. Therefore there is a positive integer $n$ and a normal subgroup $H$ such that $H \in \mathcal{L}\left(\mathcal{N}_{k}\right)$ and $|G / H|=$ $n$. So $H$ is a $(k+1)$-Engel group and $x^{n}, y^{n} \in H$ for any $x, y$ in $G$. Hence, $\left[x^{n},{ }_{k+1} y^{n}\right]=1$ and consequently $G$ belongs to $E_{k+1}^{*}$.

Proof of Theorem 1.2: Let $G$ be a finitely generated centre-by-metabelian group in $E_{k+1}^{\#}$. So $G / Z(G)$ is a finitely generated metabelian group which belongs to $E_{k+1}^{\#}$. Therefore, by [3, Theorem 1.6], $\frac{G / Z(G)}{Z_{k+1}(G / Z(G))}$ is finite; so $G / Z_{k+2}(G)$ is finite. It follows, by [6, Theorem 1], that $G$ is in the class $\mathcal{F N}_{k+2}$. Let $H$ be a finite normal subgroup such that $G / H$ is nilpotent of class at most $k+2$. If $T / H$ is the torsion subgroup of $G / H$, then $T / H$ is finite; so $T$ is finite and $G / T$ is a torsion-free finitely generated nilpotent group of class at most $k+2$ which belongs to $E_{k+1}^{\#}$. It follows, by Lemma 2.2 , that $G / T$ is in $\mathcal{L}\left(\mathcal{N}_{k}\right)$; so $G$ belongs to $\mathcal{F} \mathcal{L}\left(\mathcal{N}_{k}\right)$; as required.

Conversely, suppose that $G$ is in the class $\mathcal{F} \mathcal{L}\left(\mathcal{N}_{k}\right)$. Therefore there is a finite normal subgroup $H$ such that $G / H$ is $(k+1)$-Engel. Since $G$ is a finitely generated soluble group, $G / H$ is therefore nilpotent. It follows that $G$ is finite-by-nilpotent, so $G$ is residually finite. Consequently, there is a normal subgroup $N$ of finite index such that $H \cap N=1$. Since $G / N$ is finite, if $X$ is an infinite subset of $G$, then there are $x, y \in X$ such that $x \neq y$ and $x N=y N$. We have $\left[x, k_{k+1} y\right] \in H$ and $\frac{\langle x, y\rangle N}{N}$ is cyclic, since $G / H$ is $(k+1)$-Engel and $x N=y N$. Thus, $\left[x,{ }_{k+1} y\right] \in H \cap N$. It follows that $[x, k+1 y]=1$ and, therefore, $G$ belongs to $E_{k+1}^{\#}$. 
Now we suppose that $G$ is a torsion-free centre-by-metabelian group in the class $E_{k+1}^{\#}$ and let $x, y_{1}, \ldots, y_{k+1} \in G$. Then $H=\left\langle x, y_{1}, \ldots, y_{k+1}\right\rangle$ is a torsion-free finitely generated centre-by-metabelian group. It follows, from the first part of the proof, that $H$ belongs to $\mathcal{F} \mathcal{L}\left(\mathcal{N}_{k}\right)$, and consequently $H \in \mathcal{L}\left(\mathcal{N}_{k}\right)$ since it is torsion-free. Hence, $\left[x^{y_{1}}, \ldots, x^{y_{k+1}}\right]=1$ and, therefore, $G$ belongs to $\mathcal{L}\left(\mathcal{N}_{k}\right)$.

For the proof of Theorem 1.3, we need further lemmas. Note that it is proved in [8, Theorem 2.3] that every non-torsion $k$-Baer group is a $k$-Engel group. But the converse is shown only in the metabelian case. As a consequence of Morse's result [12], we will extend this result with the following lemma:

Lemma 2.3. Let $G$ be a non-torsion centre-by-metabelian group. Then, $G$ is a $k$-Baer group if, and only if, $G$ is a $k$-Engel group.

Proof: Let $G$ be a non-torsion centre-by-metabelian group, and suppose that $G$ is a $k$-Engel group. From [12, Theorem 2], $G$ is in $\mathcal{L}\left(\mathcal{N}_{k-1}\right)$. Let $x$ in $G$; then $x^{G}$, the normal closure of $x$ in $G$, is in $\mathcal{N}_{k-1}$. Now, it is well known that subgroups of a group of nilpotency class at most $k-1$ are subnormal of defect $k-1$. Thus, $\langle x\rangle$ is $(k-1)$-subnormal in $x^{G}$, so $\langle x\rangle$ is $k$-subnormal in $G$. It follows that $G$ is a $k$-Baer group.

Lemma 2.4. Let $G$ be a torsion-free group in $\mathcal{L}\left(\mathcal{N}_{k}\right)$. If $G$ belongs to $\mathcal{B}_{k}^{*}$, then $G$ is a k-Engel group.

Proof: Let $x, y$ in $G$; since $G$ is torsion-free, the subset $\left\{x^{i}: i\right.$ positive integer $\}$ is infinite. Therefore there is a positive integer $i$ such that $\left\langle x^{i}\right\rangle$ is $k$-subnormal in $G$. Thus, $\left[x^{i},\left[y,{ }_{k-1} x^{i}\right]\right] \in\left\langle x^{i}\right\rangle$, so $\left[x^{i},\left[y,{ }_{k-1} x^{i}\right]\right]=x^{r}$ for some integer $r$. Since $G$ belongs to $\mathcal{L}\left(\mathcal{N}_{k}\right)$, we have that $G$ is a $(k+$ 1)-Engel group. Hence, $1=\left[x^{i},{ }_{k+1}\left[y,{ }_{k-1} x^{i}\right]\right]=x^{r^{k+1}}$; and this gives that $r=0$ as $G$ is torsion-free. It follows that $\left[x^{i},\left[y,{ }_{k-1} x^{i}\right]\right]=1$, so $\left[y,{ }_{k} x^{i}\right]=1$. Now, because $x^{G}$ is in $\mathcal{N}_{k}$, we have that every commutator in $x^{G}$ of length $k$ is multilinear. Thus $1=\left[y,{ }_{k} x^{i}\right]=\left[\left[y, x^{i}\right],{ }_{k-1} x^{i}\right]=$ $\left[y,{ }_{k} x\right]^{i^{k}}$. Once again, as $G$ is torsion-free, we obtain that $\left[y,{ }_{k} x\right]=1$; this means that $G$ is a $k$-Engel group.

Proof of Theorem 1.3: Let $G$ be a finitely generated centre-by-metabelian group in the class $\mathcal{B}_{k}^{*}$. So every infinite subset of $G$ contains an element $x$ such that $\langle x\rangle$ is $k$-subnormal in $G$. Hence, for any $y$ in $G$ we have $\left[y,{ }_{k+1} x\right]=1$. Thus, $G$ belongs to $E_{k+1}^{\#}$. It follows, from [11, Theorem 1], that $G$ is finite-by-nilpotent. Therefore there is a finite normal subgroup $T$ such that $G / T$ is a torsion-free centre-by-metabelian 
group which belongs to $E_{k+1}^{\#}$. It follows from Theorem 1.2 that $G / T$ is in $\mathcal{L}\left(\mathcal{N}_{k}\right)$, and by Lemma 2.4, we obtain that $G / T$ is a $k$-Engel group. Therefore, $G$ is finite-by-( $k$-Engel); as claimed.

Now, assume that $G$ is a torsion-free centre-by-metabelian group in $\mathcal{B}_{k}^{*}$ and let $x, y$ in $G$. Then, from the first part of the proof, $H=\langle x, y\rangle$ is finite-by-( $k$-Engel). Since $G$ is torsion-free we deduce that $H$ is $k$-Engel. Hence, $\left[y,{ }_{k} x\right]=1$, so $G$ is a $k$-Engel group.

Conversely, suppose that $G$ is a torsion-free centre-by-metabelian and a $k$-Engel group. From Lemma 2.3 we get that $G$ is a $k$-Baer group, so $G$ is in $\mathcal{B}_{k}^{*}$.

\section{References}

[1] A. Abdollahi, Some Engel conditions on infinite subsets of certain groups, Bull. Austral. Math. Soc. 62(1) (2000), 141-148.

[2] A. Abdollahi and B. Taeri, A condition on finitely generated soluble groups, Comm. Algebra 27(11) (1999), 5633-5638.

[3] A. Abdollahi and N. Trabelsi, Quelques extensions d'un problème de Paul Erdös sur les groupes, Bull. Belg. Math. Soc. Simon Stevin 9(2) (2002), 205-215.

[4] C. Delizia, A. Rhemtulla And H. Smith, Locally graded groups with a nilpotency condition on infinite subsets, J. Austral. Math. Soc. Ser. A 69(3) (2000), 415-420.

[5] G. Endimioni, Groups covered by finitely many nilpotent subgroups, Bull. Austral. Math. Soc. 50(3) (1994), 459-464.

[6] P. Hall, Finite-by-nilpotent groups, Proc. Cambridge Philos. Soc. 52 (1956), 611-616.

[7] L.-C. Kappe And R. F. Morse, Levi-properties in metabelian groups, in: "Combinatorial group theory" (College Park, MD, 1988), Contemp. Math. 109, Amer. Math. Soc., Providence, RI, 1990, pp. 59-72.

[8] L.-C. Kappe and G. Traustason, Subnormality conditions in non-torsion groups, Bull. Austral. Math. Soc. 59(3) (1999), 459-465.

[9] J. C. Lennox and J. Wiegold, Extensions of a problem of Paul Erdös on groups, J. Austral. Math. Soc. Ser. A 31(4) (1981), 459-463.

[10] P. Longobardi, On locally graded groups with an Engel condition on infinite subsets, Arch. Math. (Basel) 76(2) (2001), 88-90. 
[11] P. Longobardi And M. Maj, Finitely generated soluble groups with an Engel condition on infinite subsets, Rend. Sem. Mat. Univ. Padova 89 (1993), 97-102.

[12] R. F. Morse, Solvable Engel groups with nilpotent normal closures, in: "Groups St. Andrews 1997 in Bath, II", London Math. Soc. Lecture Note Ser. 261, Cambridge Univ. Press, Cambridge, 1999, pp. 560-567.

[13] B. H. Neumann, A problem of Paul Erdös on groups, J. Austral. Math. Soc. Ser. A 21(4) (1976), 467-472.

[14] M. F. Newman, Some varieties of groups, Collection of articles dedicated to the memory of Hanna Neumann, IV, J. Austral. Math. Soc. 16 (1973), 481-494.

[15] D. J. S. Robinson, "A course in the theory of groups", Graduate Texts in Mathematics 80, Springer-Verlag, New York-Berlin, 1982.

[16] B. TAERI, A question of Paul Erdös and nilpotent-by-finite groups, Bull. Austral. Math. Soc. 64(2) (2001), 245-254.

[17] N. Trabelsi, Characterisation of nilpotent-by-finite groups, Bull. Austral. Math. Soc. 61(1) (2000), 33-38.

Département de Mathématiques

Faculté des Sciences

Université Ferhat Abbas

Sétif 19000

Algérie

E-mail address: trabelsi_dz@yahoo.fr

Primera versió rebuda el 25 de setembre de 2002, darrera versió rebuda el 19 de març de 2003. 